\title{
Risco de queda nos idosos: educaçáo em saúde para melhoria da qualidade de vida
}

\section{Risk of falling on elders: health education to improve quality of life}

\author{
Augusto Nogueira ${ }^{1}$ \\ Miriam Alberto ${ }^{2}$ \\ Geraldo de Assis Cardoso ${ }^{3}$ \\ Maria Auxiliadora Motta Barreto ${ }^{4}$
}

\begin{abstract}
Resumo
A população mundial, sobretudo, a brasileira vem envelhecendo com muita rapidez. Com isso, é de suma importância conhecer as principais causas da diminuição de qualidade de vida nessa faixa etária, sendo uma dessas causas, a queda. A queda é causada por fatores intrínsecos, ou seja, próprios do indivíduo e, por fatores extrínsecos, relacionados ao ambiente. Dessa forma identificamos, neste estudo, fatores que podem ser trabalhados por diversos profissionais agindo na prevenção primária das quedas, oferecendo educação em saúde à população e capacitando equipes para trabalhar com famílias que possuam pessoas da terceira idade. O Programa de Saúde da Família - PSF, apresenta, em geral, condiçôes ótimas para se trabalhar a prevenção das quedas, orientando as famílias quanto aos riscos ambientais da queda. O treinamento para prevenção também deve ser estendido aos profissionais médicos, enfermeiros e profissionais de apoio que precisam ser treinados a identificar e controlar fatores responsáveis pela queda. Como a longevidade aumenta progressivamente, tais atitudes ampliam e atendem à necessidade dos cuidados básicos com a saúde da população em geral.
\end{abstract}

Palavras chave: queda; idosos; fatores intrínsecos; fatores extrínsecos

\begin{abstract}
The world population, beyond, Brazilians' is getting odes quickly, and with this is extremely important to look for the main causes for the reducing quality of life at this age, being one of the fall. The fall is caused by intrinsic factor, it means, itself and, for extrinsic factor, related to the environment. Thus we have identified in this work, factors that can be worked by several professionals acting in the primary prevention of falls, providing health education to the population and enabling teams to work with families who are seniors The Family Health Program - PSF, has, in general, optimal conditions to work for the prevention of falls, advising families about the risks of falling. The prevention training should also be extended to medical professionals, nurses and support they need to be trained to identify and control factors responsible for the fall. As longevity increases progressively extend these attitudes and address the need of basic care for the health of the general population.
\end{abstract}

Key words: fall; elders; intrinsic factor; extrinsic factor.

\footnotetext{
${ }^{1}$ Especialista em Fisioterapia Cardiopulmonar pela UCB, Especialista em Fisioterapia em UTI Prática pela UCB, Especialista em Docência do Ensino Superior pela UCAM e em Saúde da Família do UniFoa.

${ }^{2}$ Especialista em Medicina do Trabalho pela USF e em Gerontologia Aplicada pela UFF e em Saúde da Família do UniFoa.

${ }^{3}$ Médico, Mestre em Educação em Saúde pelo Unifoa e Coordenador da Pós-Graduação em Saúde da Família do UniFoa.

${ }^{4}$ Psicóloga, Doutora em Psicologia como Profissão e Ciência pela PucCampinas, Mestre em Educação pelo UNISAL, docente dos cursos de pós-graduação lato e stricto sensu do UniFOA.
} 


\section{INTRODUÇÃO}

O envelhecimento da população é um fenômeno mundial que vem ocorrendo com rapidez, principalmente no Brasil. Estudos apontam que em 2020, 15\% da população brasileira terá mais de 60 anos, assim, pode-se afirmar que, segundo padrôes estabelecidos pela Organização Mundial de Saúde (OMS), o Brasil, antes denominado um país de jovens, hoje já é visto como um país estruturalmente envelhecido. (PAIXÃO et al., 2009)

Este fenômeno traz à tona a discussão sobre a qualidade de vida dos idosos e, associado a isso, eventos que incapacitam e tornam o idoso dependente, sendo que as quedas estão entre as principais causas incapacitantes do idoso. A queda pode ser conseqüência de vários fatores, sendo eles intrínsecos, ou seja, decorrente do próprio envelhecimento, ou extrínsecos, causada por fatores externos, ou seja, ambientais (LOPES et al, 2007). De acordo com Fabrício, Rodrigues e Júnior (2004) a conseqüência mais comum de uma queda sofrida por idoso é a fratura, sendo mais comum fratura de fêmur seguida pela fratura de rádio, clavícula e, as outras, como coluna, úmero, escápula, patela e nariz vêem em seqüência.

A queda pode ser considerada um caso de saúde pública e grande causa de morte em idosos. Segundo o Ministério da Saúde, 14,24\% dos idosos com 80 anos ou mais morreram depois de sofrerem quedas e entre os de 70 e 79 anos, foram 5,26\% de mortes decorrentes de quedas. No Brasil, 30\% dos idosos caem ao menos uma vez por ano, sendo que $5 \%$ das quedas resultam em fraturas e entre 5\% e 10\% levam a ferimentos que necessitam de atendimento médico. Pelo DATASUS, as internaçóes decorrentes de queda em idosos vêem aumentando ano a ano. Em 2004, foram 50.906 internaçôes hospitalares, em 2005, foram 61.368 e em 2006, foram 63.381 internações (ALVES JÚNIOR e PAULA, 2008).

Estudos indicam que $66 \%$ dos idosos sofrem quedas no próprio lar (FABRÍCIO, RODRIGUES e JÚNIOR, 2004) e as principais causas das quedas são fatores relacionados com o ambiente em $54 \%$ dos casos, seguido por doenças neurológicas em 14\% e cardiovasculares em 10\%. É importante ressaltar que em torno de $30 \%$ das pessoas com mais de 65 anos e metade das com mais de 80 anos sofrem uma queda a cada ano, como apontam Pedrinelli et al (2009).

Segundo Fabrício, Rodrigues e Júnior (2004) as quedas geram não apenas prejuízos físicos, como fraturas e mortes, mas também psicológicas, como o medo de nova queda, o que leva restrição de atividades e ao declínio de saúde. Também causam aumento dos custos com os cuidados de saúde, expressos pela utilização de vários serviços especializados e pelo aumento de hospitalizaçôes.

Com o presente artigo temos o objetivo de identificar os fatores que levam o idoso a sofrer quedas, através de re- visão de literatura, para estimular o trabalho de prevenção primária dessa situação e junto a essa população.

\section{FATORES QUE LEVAM À QUEDA}

Existem vários fatores de riscos relacionados às quedas em idosos, sendo muito difícil definir somente uma causa para essas ocorrências. Há uma interação complexa entre déficits corporais intrínsecos da pessoa (fatores intrínsecos), demandas / perigos ambientais (fatores extrínsecos), além dos aspectos relacionados à realização da ação (fatores situacionais). A maioria das quedas é a combinação de fatores da pessoa e do ambiente, sendo que $44 \%$ ocorrem na presença de perigos ambientais. As quedas por motivos não esclarecidos são mais comuns em mulheres e a sua incidência aumenta com a idade (SILVA et al, 2007).

Dentre os fatores desencadeadores das quedas vamos abordar os intrínsecos e os extrínsecos, pois os situacionais referem-se às diversas possibilidades de ação do/sobre o indivíduo.

\subsection{Fatores Intrínsecos}

Sabemos que o envelhecimento é um processo biológico, progressivo, declinante e universal, no qual é possível reconhecer marcas físicas e fisiológicas inerentes. Essas mudanças anatômicas e funcionais, inerentes ao envelhecer podem ser ou não patológicas - nesses casos são desenvolvimentais - variando de pessoa a pessoa. Algumas dessas mudanças favorecem as quedas, fazendo parte no que foi denominado de fatores intrínsecos.

Podemos citar como fatores intrínsecos não patológicos a regulação da pressão arterial, os impulsos sensoriais, a marcha e o equilíbrio e dentre os patológicos a osteoporose, acidente vascular encefálico (AVE), diabetes, depressão e as demências (REBELATTO e MORELLI, 2004).

Para Freitas et al. (2006) a postura ereta dos homens, que é característica na espécie, é conseguida de uma maneira complexa, da qual participam as vias sensitivas e motoras e é afetada com o avanço da idade. O aumento da idade é associado com a diminuiçáo do aparecimento de reflexos proprioceptivos mais lentos de posição, diminuição da força dos músculos, importantes na manutenção da postura e aumento da oscilaçáo do porte. Da mesma forma as mudanças do andar também ocorrem com o envelhecimento. No geral os idosos não levantam o pé muito alto, sendo que as mulheres levantam menos que os homens, dificultando subir ladeiras, degraus e, por conseqüência, facilitando as quedas. É característico dos homens idosos desenvolverem passos largos, com pequenas separaçóes. As mulheres idosas geralmente caminham com passadas estreitas, vacilantes. 
Há, também, marchas relacionadas com patologias, tais como a de pequenos passos quando a passada não ultrapassa mais que a metade do outro pé, sendo arrastada, pois o pé mal se eleva do chão. É notada nos casos mais adiantados de arteriosclerose cerebral. Existe, também, a claudicante, por problemas osteoarticulares, caracterizada pela pessoa colocar o pé no solo com muito cuidado, geralmente pela dor, obrigando as posturas mais variadas; a caprina, em que o membro é muito elevado e colocado com a ponta, tal como os caprinos e ocorre no alcoolismo crônico, em algumas viroses e intoxicaçóes exógenas (FREITAS et al, 2006, REBELATTO e MORELLI, 2004).

Todas as marchas alteradas podem facilitar quedas em qualquer idade, mas nos idosos são ainda mais problemáticas. Segundo Ganança et al (2006) dois órgãos participam de modo importante no controle da postura: os olhos e os ouvidos. A diminuição das percepçôes visuais e auditivas, tão comuns na velhice, facilita a queda.

No campo visual ocorre o que se denomina presbiopia: alteraçôes nos tecidos conjuntivos levam à menor distensibilidade na íris e no cristalino, estreitando a circunferência do orifício pupilar, problemas de acomodação e ligeiras mudanças na percepção da cor. Tudo isso leva a uma diminuição da capacidade de ver claramente os objetos, focalizar a distâncias diferentes, funcionar com pouca luz, discernir a intensidade das cores e avaliar distâncias (GANANÇA et al, 2006)

A visão provê informaçóes sobre o movimento da cabeça e dos objetos ao redor, sendo uma importante sinalizadora das características do ambiente, o que transforma em fonte fundamental de informação. A diminuição da acuidade visual, principalmente para perto, pode fazer com que o idoso não perceba detalhes como degraus, abjetos no chão, facilitando quedas. A diminuição do campo visual periférico e superior faz com que os idosos só percebam os objetos entrando no campo de visão quando estấo muito perto, esbarrando em quinas de mesa, poltronas, quaisquer objetos e móveis, podendo se ferir ou cair mesmo em ambientes aos quais está acostumado. Da mesma forma, a lentidão de adaptação do olho no escuro pode facilitar quedas noturnas dos idosos ao irem ao banheiro à noite, o que ocorre com bastante freqüência.

A diminuição de noção de profundidade, da capacidade de estimar a distância relativa e prever objetos e obstáculos, assim como a diminuição de discriminação de cores, principalmente no espectro azul-verde e da capacidade de lidar co ofuscamento quando a luz reflete em superfícies polidas, podem facilitar as quedas quando os idosos se encontram em ambientes com monotonia de cores, por exemplo, paredes e janelas brancas ou em dias com sol muito intenso, refletindo em pisos, objetos ou mesmo em lâminas de água (GANANÇA et al, 2006).
A perda auditiva pode ser o marcador grosseiro de fragilidade do idoso, havendo dificuldade de perceber sons com intensidade alta no começo indo para os de média e baixa, levando a uma menor compreensão de vários sons no meio ambiente. Isso pode torná-lo ansioso, angustiado, depressivo, instável, facilitando as quedas. O sistema vestibular é referência interna sobre os limites da estabilidade e controle postural, ocorrendo alteraçóes na velhice, levando a um aumento do tempo de latência da resposta do ajuste postural, dificultando recuperar o centro do corpo em movimentos bruscos, tais como tropeçôes e escorregôes.

De acordo com Freitas et al (2006), o envelhecimento leva a modificaçôes nos tecidos musculares, conjuntivo e ósseo levando a uma diminuição da força, elasticidade, amplitude nos movimentos e trazendo dificuldade para os idosos levantarem de locais baixos como sofás e vasos sanitários.

Outro fator intrínseco que deve ser considerado é o estado do sistema nervoso, que constitui-se no principal fator de integração das atividades orgânicas e é fundamental para a manutenção da homeostase. Os centros reguladores localizam-se no cérebro que no homem de 70 anos de idade tem uma redução de 5\% de seu peso, e aos 90 anos, de 20\%. A atrofia é observada tanto na camada cortical quanto na substância branca. Os neurônios no sistema nervoso central comunicam-se através de sinapses, onde são liberadas substâncias denominadas neurotransmissores, responsáveis pela passagem dos estímulos de um neurônio ao outro. Há diminuição da liberação dos neurotransmissores no envelhecimento. (SILVA et al, 2007, REBELATTO e MORELLI, 2004).

Os centros reguladores sáo estimulados por receptores que se localizam em vários pontos do organismo. Ocorre diminuição da sensibilidade dos barorreceptores ou receptores de pressão que se localizam principalmente nas paredes da aorta e das artérias carotídeas, provavelmente devido à diminuição da elasticidade da parede. $\mathrm{O}$ mesmo acontece com os quimiorreceptores localizados na crosta aórtica e nas artérias carotídeas internas e no sistema nervoso central, e que são suscetíveis às variações das pressóes de oxigênio e gás carbônico e do $\mathrm{PH}$.

Tudo isso leva a uma lentidão nas respostas motoras e tendência a modificação do tônus postural, alterando o equilíbrio do idoso sendo causa ou facilitador das quedas.

Além disso, diversas condiçóes patológicas que aumentam em prevalência ao envelhecer podem contribuir para a instabilidade e a queda. A doença degenerativa das articulaçóes (especialmente as cervicais, lombossacras e de membros inferiores) pode causar dores e instabilidades motoras (SILVA et al, 2007).

Para Hamra, Ribeiro e Miguel (2007) a osteoporose é uma enfermidade inicialmente silenciosa, de grande importância em saúde pública, que vai diminuindo a massa óssea, acarretando a insuficiência mecânica ou falência do osso, 
facilitando as quedas. Além de elevada morbidade, é responsável por grande incidência de hospitalizações e óbitos. Estudos de freqüência mostram que, de cada três mulheres entre 45 e 80 anos, uma apresenta sinais de osteoporose e nos homens, é de uma para cinco.

As manifestaçôes das fraturas por osteoporose podem se silenciosas como as que ocorrem nas vértebras torácicas, ou muito dolorosas, e serem precipitadas por um esforço pequeno como sentar-se. Tanto a dor como a insegurança e o medo podem favorecer uma queda e sempre há dúvida se a fratura no fêmur, por exemplo, aconteceu devido à queda, ou se a queda aconteceu porque já havia a fratura por um trauma pequeno em um fêmur osteopênico. Da mesma forma, a hipotensão ortostática ou postural ocorre em cerca de $20 \%$ dos idosos, estando associada a vários problemas nesta população, incluindo fraqueza ou atordoamento ao ficar de pé, uso de medicamentos diversos, podendo ser a causa da queda. (PERRACINI, 2005)

Além disso, segundo Ribeiro et al (2008), muitos processos de doenças, especialmente as cardiovasculares e do sistema neurológico-cognitivo podem ser associadas às quedas: arritmias cardíacas, acidente vascular cerebral, doença de Parkinson, depressão, demência.

Os idosos apresentam um risco maior no uso de medicamentos, já que os consomem mais freqüentemente, em maior diversidade, e muitas vezes, ao mesmo tempo, podendo maximazar efeitos colaterais. Para adequado manejo das drogas em idosos é fundamental que sejam consideradas as alteraçôes fisiológicas próprias do envelhecer, já que a farmacocinética, de vários medicamentos, pode ser influenciada por isso. A preferência deve ser dada às drogas com menos efeitos colaterais, cujas doses devem ser ajustadas segundo a resposta e a tolerabilidade do paciente.

É importante considerar todos os medicamentos usados, uma vez que $75 \%$ dos idosos tomam medicamentos por conta própria, que causam ou contribuem para sintomas e interagem com outros. Não se deve esquecer, também, da possibilidade do abuso de substâncias tóxicas como maconha, cocaína, álcool e outras, que idosos podem continuar a usar, favorecendo as quedas (RIBEIRO et al, 2008).

\subsection{Fatores Extrínsecos}

Os fatores extrínsecos responsáveis por queda em idosos são constituídos pelos riscos ambientais, os riscos durante a realizaçáo das atividades de vida diária e, em idosos com maior imobilidade os movimentos como virar-se no leito, sentar-se e levantar-se. Porém, a maior parte das quedas ocorre durante atividades de rotina. (FREITAS et al, 2006). De acordo com estudo feito por Ganança et. al (2006) as tarefas mais realizadas na hora da queda são a deambulação, subir ou descer escadas, mudança de postura e ao tomar banho.
É comum que o idoso passe a maior parte do tempo em seu domicílio e, por ser um ambiente familiar, pode se tornar muitas vezes um lugar de risco, pois a autoconfiança causada pelo conhecimento do local diminui a atenção. Já em local externo, apesar de trazer mais risco, é comum o idoso permanecer por um tempo pequeno e respeitar seus obstáculos (SILVA et al, 2007).

Segundo Lopes et al (2007) os riscos ambientais são os que mais ocasionam queda nos idosos, sendo que a maioria das vezes a queda ocorre no ambiente doméstico sendo mais comum em escadas, no quarto, na sala e no banheiro. Estudos apontam que os homens caem mais no quarto, enquanto as mulheres caem igualmente nas diferentes partes da casa.

Os fatores extrínsecos são potencialmente influenciados pelos fatores ambientais, tais como, iluminação inadequada, superfícies escorregadias, tapetes altos ou estreitos, obstáculos, calçados e roupas inadequadas, maus-tratos, buracos e irregularidades no solo (ALVES JÚNIOR e PAULA, 2008).

Para Ganança et al (2006) as condiçôes do ambiente que impóe maior risco para queda são a presença de tapete, o chão úmido e a presença de degraus. Já de acordo com trabalho feito por Lopes et al (2007) as causas predominantes das quedas de idosos são fatores ambientais como piso molhado, apontado como principal causa dos acidentes em pesquisa realizada. Segundo os participantes do trabalho, as quedas ocorreram predominantemente no banheiro e na cozinha. Da mesma forma, em estudo realizado por Ribeiro et al (2008) as maiores causas de queda em idosos são o piso escorregadio na casa e no banheiro, calçados inadequados e degraus na soleira da porta.

Em pesquisa descrita por Hamra, Ribeiro e Miguel (2007) onde 205 idosos que sofreram queda responderam a um formulário, $74,6 \%$ das quedas ocorreram no ambiente doméstico, sendo os locais mais freqüentes: quintal (19\%), banheiro $(18,5 \%)$, sala $(12,2 \%)$, quarto $(7,8 \%)$ e cozinha (7,8\%). Em relaçáo ao período do dia, 46,3\% dos idosos que responderam ao formulário caíram no período vespertino, 39\% no matutino e $14,6 \%$ no noturno. Porém Ganança et al (2006) encontraram em outro trabalho com 64 idosos que sofreram quedas, que no período matutino houve $51,6 \%$ das quedas, de tarde ocorreram $34,4 \%$, de noite foram $12,5 \%$ e de madrugada aconteceu 1,6\% das quedas.

Para Veras et. al (2007) identificar os fatores de risco ambientais é essencial para prevenir problemas mais graves que possam dificultar ou impedir a realização das atividades de vida diária do idoso.

É importante ressaltar que algumas causas intrínsecas como a diminuiçáo do estímulo sensorial, perda de mobilidade ou deformidades, aumentam o risco de queda quando associadas a fatores de risco ambientais, tais como a baixa iluminação do ambiente ou brilho excessivo (FREITAS et 
al., 2006). A perda da força muscular e episódios de vertigem podem proporcionar maior risco quando associados à atividade de vida diária como se virar rapidamente, descer de um banquinho ou usar o banheiro. Segundo Freitas et al. (2006) pessoas com diminuição da mobilidade e do controle postural podem sofrer quedas durante atividades simples como passar da cama para a cadeira ou inclinar-se.

\section{CONSIDERAÇÓES FINAIS}

A queda em idosos é uma das principais causas de óbito ou de incapacitação, gerando grande gasto do governo com hospitalização. Além dessas conseqüências concretas, práticas, existem grandes desdobramentos emocionais e psíquicos para o idoso que cai e sua família. Porém as quedas podem ser evitadas ou diminuídas na grande maioria dos casos, com medidas simples.

Primeiro é importante trabalhar sobre as causas intrínsecas que levam o idoso a cair e, para isso, é importante a atuação da família e de uma equipe multidisciplinar. Deve-se sempre verificar a visão e ficar atento a qualquer diminuição ou alteração auditiva. Se o idoso passa a ficar sonolento é importante verificar a causa, que pode estar relacionada a uso de medicamentos ou algum distúrbio, como o de tireóide. Também é necessário amenizar a ação de possíveis comorbidades como seqüela de AVE, deformidades articulares e ósseas, desvios posturais, disfunção do sistema vestibular, incontinência urinária que leva a levantar várias vezes à noite, diabetes e hipertensão arterial, entre outros.

Concomitantemente aos fatores intrínsecos das quedas, é necessário estar atento aos fatores extrínsecos. É imprescindível eliminar qualquer risco que exponha o idoso à queda no ambiente onde ele vive e, para isso, analisar o ambiente no qual ele habita é uma das principais fontes de informação. Assim deve-se eliminar tapetes da casa que possam levar o idoso a escorregar ou tropeçar, eliminar móveis que ficam na altura das pernas e no meio dos ambientes, manter o piso sempre seco e sem produtos que possam deixá-lo escorregadio, manter a casa iluminada e sem brilho excessivo, regular a altura dos assentos e da cama assim como do vaso sanitário, para que se tenha acesso aos mesmos sem grandes esforços musculares. Também é importante orientar o idoso quanto ao calçado ideal e cuidados com o uso de órteses para auxílio da marcha.

A queda, como um acontecimento multifatorial deve ter seu foco não apenas na prevenção dos fatores de risco ambientais, mas também nos hábitos e nas atividades de vida diária - AVD - dos idosos, que tomam atitudes ou realizam atividades que podem apresentar riscos.
No Programa de Saúde da Família - PSF, existem condiçóes ótimas para se trabalhar a prevenção das quedas, considerando ser necessária a capacitação dos Agentes Comunitários de Saúde para orientar as famílias quanto aos riscos ambientais da queda. O treinamento para prevenção também deve ser estendido aos profissionais médicos, enfermeiros e profissionais de apoio que precisam ser treinados a identificar e controlar os fatores intrínsecos responsáveis pela queda.

Considerando que a longevidade aumenta progressivamente, tais atitudes ampliam e atendem à necessidade dos cuidados básicos com a saúde da população em geral. 


\section{REFERÊNCIAS:}

1. ALVES JUNIOR, Edmundo de Drummond; PAULA, Fátima de Lima. A prevenção de quedas sob o aspecto da promoção da saúde. Fitness and Performance Journal, Rio de Janeiro, vol. 7, p. 123-129, 2008.

2. FABRÍCIO, Suzele Cristina Coelho; RODRIGUES, Rosalina A Partezani; JÚNIOR, Moacyr Lobo da Costa. Causas e conseqüências de quedas de idosos atendidos em hospital público. Revista de Saúde Pública, vol. 38, n.1, São Paulo, Fev, 2004.

3. FREITAS, Elizabete Viana de; PY, Lígia; CANÇADO, Flávio Aloízio Xavier; DOLL, Johannes; GORZONI, Milton Luiz. Tratado de Geriatria e Gerontologia. 2. ed. Rio de Janeiro: Guanabara Koogan, 2006.

4. GANANÇA, Fernando Freitas; GAZZOLA, Juliana Maria; ARATANI, Mayra Cristina; PERRACINI, Monica Rodrigues; GANANÇA, Mauricio Malavasi. Circunstâncias e conseqüências de quedas em idosos com vestibulopatia crônica. Revista Brasileira de Otorrinolaringologia, vol. 72, n.3, São Paulo. Maio/Junho, 2006.

5. HAMRA, Alberto; RIBEIRO, Marcelo Barbosa; MIGUEL, Omar Ferreira. Correlação entre fratura por queda em idosos e uso prévio de medicamentos. Acta Ortopédica Brasileira. São Paulo, vol 15, n.3, p.143145, 2007.

6. LOPES, Mislaine C. de Lima; VIOLIN, Mara Rúbia; LAVAGNOLI, Ana Paula; MARCON, Sônia Silva. Fatores desencadeantes de qiedas no domicilio em uma comunidade de idosos. Cogitare enfermagem, Paraná, vol.12, n. 4, p. 472-477, out/dez. 2007.

7. PAIXÃO, Idalina Alves de Castro; NOBREGA, Fátima Rezende Gomes de; SILVA, Cláudia Forlin da; BRAGA, Teresa Cristina Lima. A inserção social do idoso através de exercício físico. Nursing, São Paulo, vol 12, n.136, p. 424-428, set. 2009.

8. PEDRINELLI, André; GARCEZ-LEME, Luiz Eugênio; NOBRE, Ricardo do Serro Azul. O efeito da atividade física no aparelho locomotor do idoso. Rev. bras. ortop., São Paulo, v. 44, n. 2, abr. 2009 . Disponível em $<$ http://www.scielo.br/scielo. php? script=sci_arttext\&pid=S0102=36162009000200002-\&lng=pt\&nrmiso>. acessos em 27 mar. 2013. http://dx.doi. org/10.1590/S0102-36162009000200002
9. PERRACINI, Monica Rodrigues. Prevenção e Manejo de Quedas. In: Ramos, LR; Toniolo Neto, J. (Org.). Guia de Geriatria e Gerontologia. São Paulo: Manole, 2005, vol. 1, p. 193-208.

10. REBELATTO, José Rubens; MORELLI, José Geraldo da Silva. Fisioterapia Geriátrica: A prática da assistência ao idoso. São Paulo: Manole, 2004.

11. RIBEIRO, Adalgisa Peixoto; SOUZA, Edinilsa Ramos de; ATIE, Soraya; SOUZA, Amaro Crispim de; SCHLITHZ, Arthur Orlando. A influência das quedas na qualidade de vida dos idosos. Ciênc. Saúde coletiva, Rio de Janeiro, vol.13, n. 4, Jul/Ago. 2008.

12. SILVA, Tatiana Magalhães; NAKATANI, Adélia Yaeko Kyosen; SOUZA, Adenícia Custódia Silva e; LIMA, Maria do Carmo Silva. A vulnerabilidade do idoso para as quedas: análise dos incidentes críticos. Revista Eletrônica de Enfermagem, vol.9, n. 1, p. 64-78, 2007.

13. VERAS, Renato Peixoto; CALDAS, Célia Pereira; COELHO, Flávia Dantas; SANCHEZ, Maria Angélica. Promovendo a Saúde e Prevenindo a Dependência: identificando indicadores de fragilidade em idosos independentes. Revista Brasileira de Geriatria e Gerontologia Rio de Janeiro, vol.10, n. 3, 2007. 